\title{
MEASUREMENT OF PRESSURE CHANGES IN THE RIGHT VENTRICLE BY CARDIAC PUNCTURE
}

\author{
BY JOHN SHAW DUNN \\ (From the Physiological Department, R.E. Experimental Station, Porton)
}

THE methods which are usually employed for registering fluctuations in the endocardial pressure entail serious operative measures of a nature which renders successive examinations in the same animal difficult or impossible. In the present communication it is proposed to describe a simple procedure whereby a series of records of endocardial pressure may be obtained at intervals extending over several days without endangering the life of the animal. The method has the special advantages that no anaesthetic is required, operative measures are reduced to a minimum, and artificial respiration is dispensed with. Between the examinations the animal leads a practically normal existence. The method as it has been employed comprises the use of a tambour and lever for recording, so that it is applicable to the study of gross alterations in the general pressure rather than to accurate registration of the rapid changes which occur during each cardiac cycle.

Goats have been used exclusively in the experiments, and have been found very suitable for various reasons. They are of comparatively large size (20-30 kilos), and the position of the heart is easy to define accurately: they are also easy to handle and exhibit great indifference to minor operative measures. An experiment is carried out in the following way: The goat is laid on its right side on the operating table. A 5 c.c. Record syringe is fitted with a needle of about $0.75 \mathrm{~mm}$. bore and $1 \frac{3}{4}$ to 2 inches long: the syringe and needle are washed out with a 10 per cent. solution of sodium citrate, and the bore of the needle remains filled with this fluid. The needle is thrust through the skin and thoracic wall until it touches the heart, and is then pushed tbrough the cardiac muscle into the cavity. In a normal goat of average size the point selected for puncture is in the third left interspace, about three finger-breadths or a little less from the anterior aspect of the sternum, and the needle is directed vertically. In the great majority of instances the right ventricle is thus easily reached at a depth of $1 \frac{1}{4}$ to $1 \frac{1}{2}$ inches. The success of the puncture is ascertained by gently withdrawing the piston of the syringe, when venous blood ascends into the barrel. The syringe is then detached from the needle and the free end of a piece of

IQ. J. M., Oct., 1919.] 
pressure tubing, filled with 10 per cent. citrate, is slipped over the needle boss. The other end of the rubber tube, which is $40 \mathrm{~cm}$. in length, is attached to the tambour chamber of a Hürthle manometer, also filled with the citrate. When the tap of the tambour is opened the lever oscillates in response to the cardiac pulsations and the oscillations are recorded on smoked paper on a revolving drum. After a tracing has been taken it is necessary to draw a zero line : this is obtained by detaching the rubber tube from the needle, the latter having been withdrawn from the heart, and holding the free end of the tube at the level of the needlepoint when the latter was in the ventricle. From the zero line a scale can then be built up by raising the free end of the rubber tube to successive distances of $5,10,15, \& c ., \mathrm{cm}$. above the zero. As the tube is raised the tambour lever rises in response to the rise of pressure and the various pressures in c.c. of citrate are marked on the tracing. From this scale it is possible later to estimate the pressure in mm. of mercury corresponding to any point on the tracing or to calculate mean systolic or diastolic pressures. It has been customary to make a scale for each separate tracing in order to eliminate fallacies which might arise from changes in the tambour membrane.

A tracing of the respiration is taken simultaneously with the blood-pressure record. The apparatus used for this is a rubber football bladder bandaged on to the thorax of the goat and connected to a recording tambour. This shows the rate and time incidence of the respirations, but gives no information as to depth. The beats of the time-marker on each record indicate seconds.

The procedure of puncturing the heart as outlined above can be carried out very readily and successfully in the great majority of normal goats. Difficulty arises in animals which struggle, as some do even when placed on the operating table. It is best to avoid the use of such. Struggling, however, is most likely to occur just when the skin and the parietal pleura are punctured, and it is then advantageous to wait a few seconds before pushing the needle farther. The heart itself appears to be insensitive to the needle.

There are two important accidental lesions which may be produced in attempting to reach the ventricle, and one or other is certain to occur from time to time, even with the best technique. One is pneumothorax, caused by tearing the edge of the lung: this is never fatal to the life of the animal, but it may interfere with the object of the experiment for other reasons. The other is haemopericardium, due to injury of a branch of a coronary vessel: bleeding apparently never comes through the puncture from the ventricle itself. The risk of haemopericardium is minimized by pushing the needle-point sharply through the muscular wall as soon as the cardiac surface is reached: damage is done if the point scrapes the visceral pericardium. Both accidents are most liable to occur if there is struggling, but neither has proved a frequent or serious complication in the normal goat.

A typical normal tracing is shown in Fig. 1. The waves corresponding to each systole occur at regular intervals and are of approximately equal height. In each the rise is steep and the summit is sustained in the form of a plateau. 
It is evident that the pulse and respiration are very little disturbed by the operation of passing the needle into the heart. The respiratory undulations are visible on the blood-pressure tracing, each inspiration being accompanied by a slight fall of systolic and diastolic pressure. Themean systolic pressure herecorresponds to $22.0 \mathrm{~mm}$. of mercury, the maximun being 23.0 and the minimum $21.0 \mathrm{~mm}$.

A record taken with the drum moving at a rapid rate gives systolic waves of the same height. This tracing brings out the fact that during almost the whole of diastole the line is truly horizontal. If the curve were damped by friction in the needle or inertia in the system one would not obtain a tracing of this type. We conclude, therefore, that the lowest point of the tracing represents the lowest pressure which exists in the right ventricle and that the summit of the tracing gives an accurate reading of the systolic pressure.

Tracings taken from fifteen normal goats have given blood-pressure curves very similar in general outline to that depicted in Fig. 1 , but there are individual variations in the average pressures, and in the extent to which the pressures are influenced by respiration. The mean pressures and the maxima and minima are given in the following table:

\begin{tabular}{|c|c|c|c|c|c|c|c|}
\hline \multirow[b]{2}{*}{ Goat No. } & \multirow[b]{2}{*}{$\begin{array}{l}\text { Resp. } \\
\text { Rate. }\end{array}$} & \multicolumn{3}{|c|}{ Systolic Pressure. } & \multicolumn{3}{|c|}{ Diastolic Pressure. } \\
\hline & & Mean. & Maximum. & Minimum. & Mean. & Maximum. & Minimum. \\
\hline D 28 & 24 & $14 \cdot 0$ & $22 \cdot 0$ & 10.0 & 1.0 & 5.0 & -4.0 \\
\hline D 25 & 16 & $15 \cdot 5$ & 16.5 & 12.5 & 1.0 & $4 \cdot 5$ & -4.0 \\
\hline D 37 & 24 & $15 \cdot 5$ & 17.0 & $14 \cdot 0$ & 1.5 & 4.5 & $-\mathbf{2 . 0}$ \\
\hline D 39 & 18 & 17.5 & $19 \cdot 0$ & 15.0 & 2.0 & 4.5 & -1.0 \\
\hline D $22 \mathrm{~b}$ & 22 & $9 \cdot 5$ & 13.0 & $7 \cdot 0$ & 2.5 & 6.0 & 0.0 \\
\hline D 42 & 22 & $15 \cdot 5$ & 17.0 & $13 \cdot 0$ & $2 \cdot 5$ & 5.5 & 0.0 \\
\hline D 35 & 22 & 19.0 & $23 \cdot 5$ & 15.0 & $3 \cdot 0$ & 8.0 & -1.5 \\
\hline D $39 \mathrm{a}$ & 16 & 22.0 & 230 & 21.0 & $5 \cdot 0$ & 6.0 & 4.0 \\
\hline D 30 & 22 & 23.5 & 26.0 & 16.0 & $5 \cdot 0$ & 9.5 & 1.0 \\
\hline D 27 & 14 & 21.5 & $22 \cdot 0$ & 19.5 & 5.5 & 6.0 & 4.5 \\
\hline D 31 & 26 & 15.0 & 17.0 & 13.0 & 5.5 & 6.0 & 1.0 \\
\hline D 40 & 34 & 18.0 & 19.5 & 17.0 & 5.5 & 6.0 & 4.0 \\
\hline D 26 & 13 & 21.5 & $26 \cdot 0$ & 20.0 & 6.0 & 8.5 & $4 \cdot 0$ \\
\hline D 36 & 16 & 22.0 & 26.5 & 16.5 & 6.0 & 12.5 & 4.0 \\
\hline D 23 & 18 & 27.5 & $29 \cdot 0$ & $26 \cdot 0$ & 11.5 & 15.0 & 8.5 \\
\hline
\end{tabular}

The mean systolic pressure, which is taken to represent the mean pressure in the pulmonary artery, varies in different animals between the extremes of 9.5 and $27.5 \mathrm{~mm}$. of mercury, and the average for the fifteen is $18.5 \mathrm{~mm}$. Thirteen out of the fifteen lie between 14.0 and $23.5 \mathrm{~mm}$. The goat with the low figure of $9.5 \mathrm{~mm}$. gave $11.0 \mathrm{~mm}$. on another examination, indicating an habitually low pressure. The highest pressure observed, $27.5 \mathrm{~mm}$, appears to bave been exceptional even in the animal which showed it, for on other occasions it had a pressure of 16.5 and $20 \mathrm{~mm}$. The curves from which these pressures have been calculated generally show flat-topped waves with a well-defined limit to each plateau, as in Fig. 1, so it may be safely assumed that there is no overthrowing of the tambour lever and that the pressures given do not err by being too high.

'The mean pressure in the pulmonary artery collected by Tiegerstedt amounts 
in the rabbit to $12 \mathrm{~mm}$, in the cat to $18 \mathrm{~mm}$, and in the dog to $20 \mathrm{~mm}$.' (Fuhner and Starling, Journ. Phys., xlvii, p. 288).

The results obtained from the unanaesthetized goat correspond, therefore, to those given above for the dog and cat, and are slightly higher than the average (14 mm. mercury) obtained by Fuhner and Starling in the heart-lung preparation. Their statement, however, that the pressure in the pulmonary artery is one-sixth of the systemic arterial pressure is substantially confirmed.

The mean diastolic, like the mean systolic pressures, exhibit a considerable range of variation, from 1.0 to $11.5 \mathrm{~mm}$., but are always above zero level. The average for the fifteen animals is $4.2 \mathrm{~mm}$. of mercury. In five out of fifteen the minimum diastolic pressure falls below zero, and in two of these the negative pressure amonnts to $4.0 \mathrm{~mm}$., which is probably well outside the range of experimental error. The negative pressures occur in diastolic periods which coincide with the rise of inspiration, when the intrathoracic pressure is lowest and the ventricular well relaxed.

No reason has been found to account for the variations in the mean pressures in different animals. From the table it appears that the lower diastolic pressures do not run parallel with the higher rates of respiration, though this appeared a probable relationship. Negative diastolic pressures have been much more frequently observed in animals which were breathing rapidly and forcibly as a result of experimental lesions.

The mean diastolic pressure in the right ventricle may be assumed to differ but little from the pressure in the large veins, as there is no intervening valve. The blood-pressure in the external jugular vein has been actually measured in sixteen normal goats and the results are set out below for comparison. The measurements were made by a U-manometer filled with 10 per cent. citrate solution, and the vein was reached by puncturing through the skin with a wide-bore needle. The pressures observed in these animals were as follows:

$\begin{array}{cc}\text { Number of Goats. } & \text { Venous Pressure in mm. Hg. } \\ 1 & 2 \cdot 0 \\ 5 & 3 \cdot 0 \\ 3 & 3 \cdot 5 \\ 4 & 4 \cdot 5 \\ 1 & 5 \cdot 0 \\ 2 & 6 \cdot 0 \\ \text { I'otal } 16 & \text { Mean } 3 \cdot 9 \mathrm{~mm} .\end{array}$

The mean pressure, $3.9 \mathrm{~mm}$., obtained by this method compares with $4.2 \mathrm{~mm}$. observed in the ventricle in the filteen other animals, and the range of variation is fairly similar. The citrate manometer allows of prolonged observation and of very accurate mcasurements, so that these figures help to confirm the approximate correctness of the pressures observed in the ventricle by cardiac puncture.

Normal tracing. In the normal tracing shown in Fig. 1 the respiratory curve is not precisely above the cardiac curve. The ares at the commencement of the tracings show the relative positions of the writing-points when the drum is at rest. Taking this fact into consideration it appears that the lowest point on the 


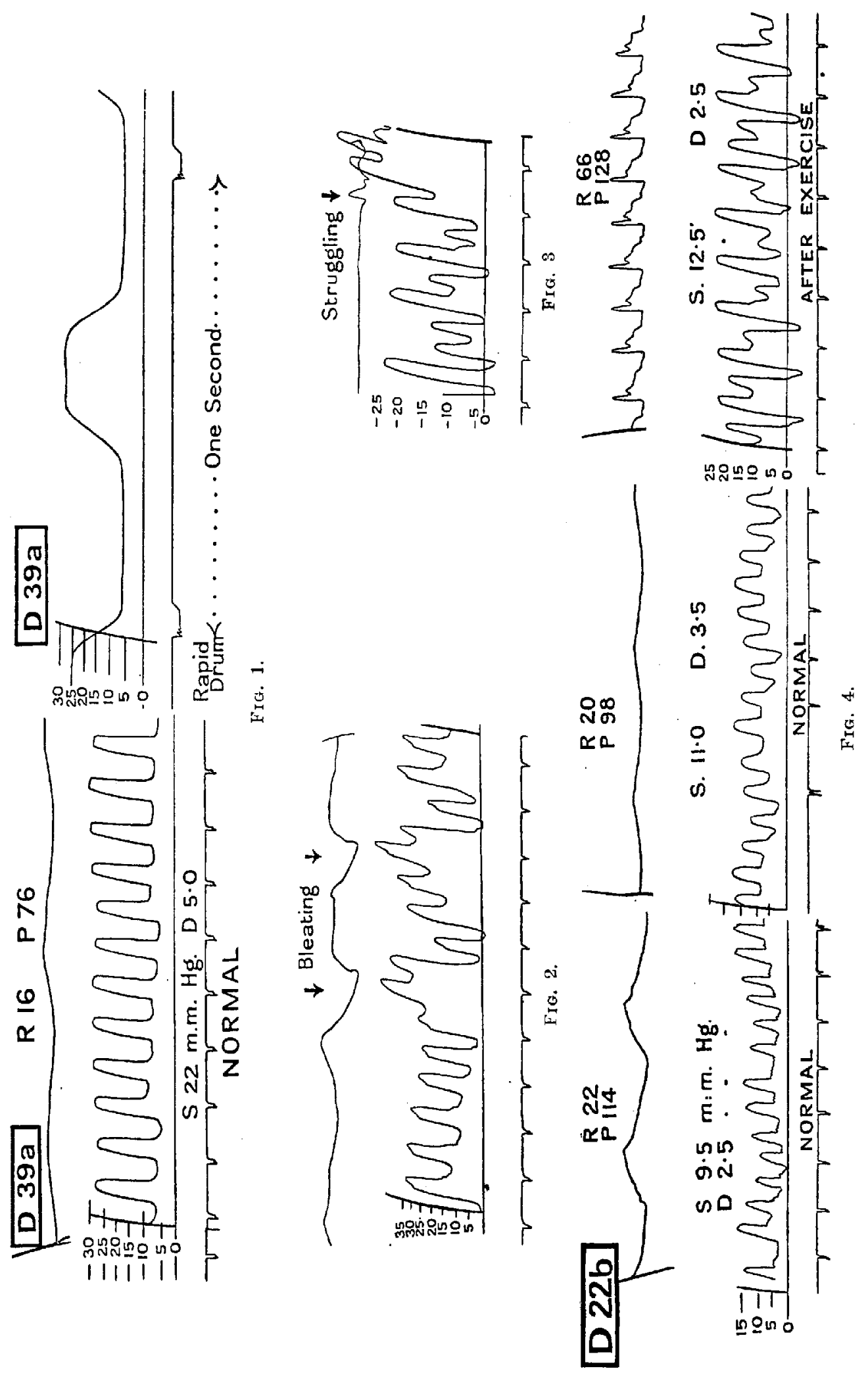


curve of both the systolic and diastolic pressures is synchronous with the height of inspiration. This fact is in contrast with the conditions on an arterial bloodpressure curve, where the height of inspiration takes place appreciably before the lowest point is reached on the respiratory undulation of the blood-pressure. It would appear, therefore, that the lag shown on the systemic blood-pressure curve is not due to any event communicated through the heart, such as, for instance, the forcing of blood into the great veins by increased pressure in the abdomon, but is due to events taking place in the thorax itself.

Effect of exercise. Measurements of the ventricular pressure were made in two goats before and immediately after exercise. The animals were induced to run twice up and down a steep gradient about seventy yards long: this was only accomplished after considerable difficulty, the result being that the goats were much less exercised than the experimenters. One of the tracings, however, shows a considerable acceleration of the respiration (Fig. 4). The first two sections are from the normal resting animal, and show the usual characters, although the mean systolic pressures are unusually low. The minimum diastolic pressure in one reaches the zero line, but does not fall below it. After exercise the respiration has risen to 66 per min. from $22 / 20$, and the pulse to 128 from $114 / 98$.

There is a remarkable change in the form of the blood-pressure curve, due to the alteration in cardio-respiratory ratio. The inspirations occur a little more than half as rapidly as the systoles, so that the influence of each inspiration falls successively a little earlier on every second cardiac cycle. The effect of this is best observed by drawing a line through the apex of every second systole: this line exhibits a slow regular undulation or 'beat' such as results from interference between any two series of vibrations which have nearly but not quite the same amplitude.

The mean, maximal, and minimal pressures in the three sections of the tracing are as follows :

\begin{tabular}{|c|c|c|c|c|c|c|}
\hline & & Systole. & & & Diastole. & \\
\hline & Mean. & Maximum. & Minimum. & Mean. & Maximum. & Minimum. \\
\hline $\begin{array}{l}\text { Normal } \\
\text { Exercise }\end{array}\left\{\begin{array}{l}(1) \\
(2)\end{array}\right.$ & $\begin{array}{r}9.5 \\
11.0 \\
12.5\end{array}$ & $\begin{array}{l}13 \cdot 0 \\
12 \cdot 5 \\
18 \cdot 0\end{array}$ & $\begin{array}{l}7 \cdot 0 \\
8 \cdot 5 \\
8 \cdot 0\end{array}$ & $\begin{array}{l}2.5 \\
3.5 \\
2.5\end{array}$ & $\begin{array}{l}6.0 \\
6 \cdot 0 \\
8 \cdot 0\end{array}$ & $\begin{array}{r}0.0 \\
1.5 \\
-4.0\end{array}$ \\
\hline
\end{tabular}

After exercise the range between maximal and minimal pressures is considerably extended, and the minimum diastolic pressure is below zero. This negative pressure is produced at points where diastole coincides with inspiration, so that the strongest negative intrathoracic pressure is acting on a relaxed ventricular wall. In spite of these greater fluctuations of pressure it will be observed that the mean systolic and diastolic pressures are hardly altered from the normal.

In the other exercise experiment (Fig. 5) the acceleration of respiration is much less, and the only change in the tracing is a slight accentuation of the respiration wave on the blood-pressure curve; the mean pressures are hardly affected. 

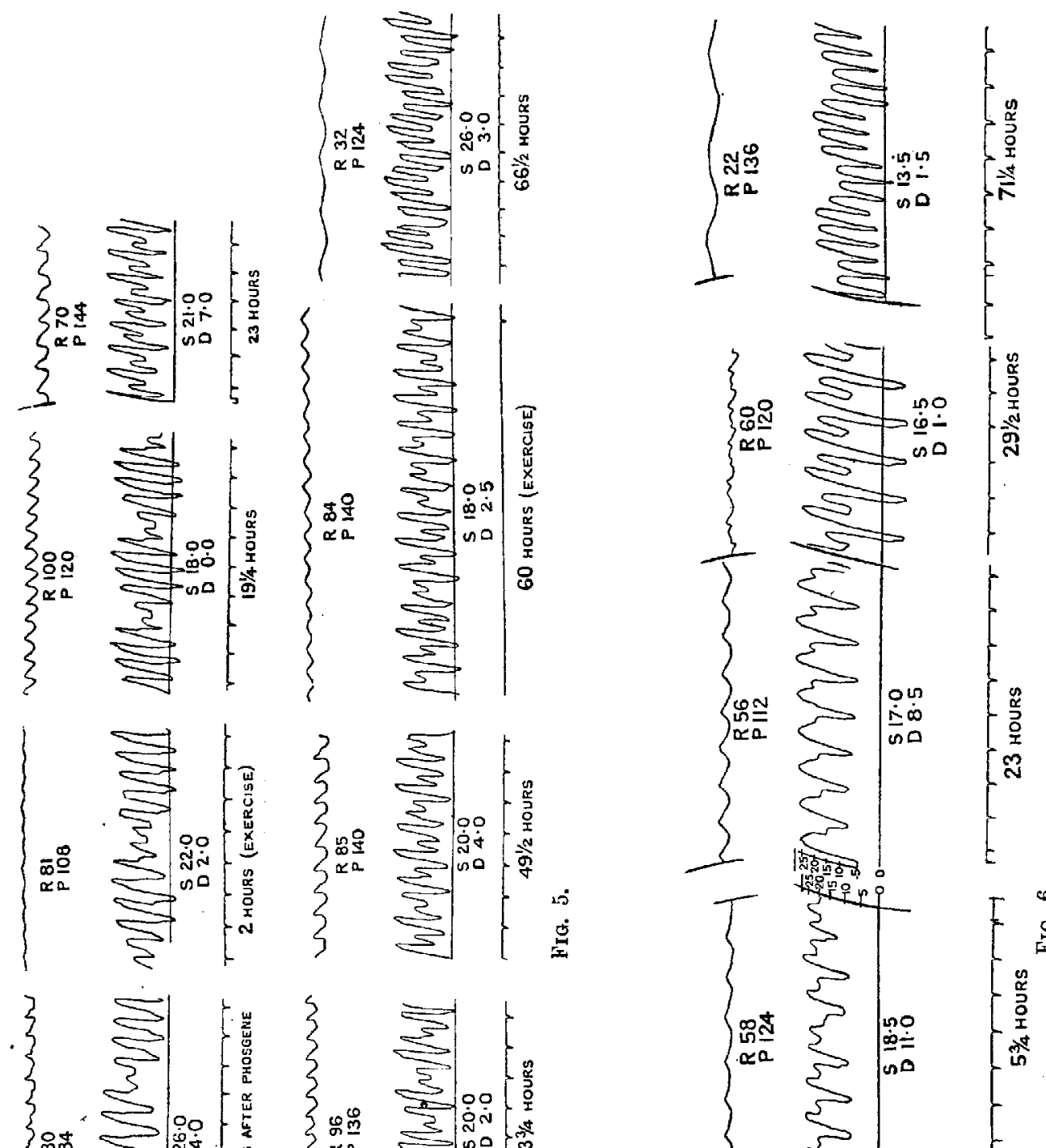
Effects of bleating and struggling. When struggling occurs while a tracing is being taken there is an immediate and very marked alteration in the bloodpressure curve. Examples of this are given in Figs. 2 and 3 . Fig. 2 shows the effect of bleating: the animal was normal when it was under examination, and gave mean pressures of $17.0 \mathrm{~mm}$. systolic, and $5.5 \mathrm{~mm} . \mathrm{Hg}$ diastolic. During the periods when the animal made the violent expiratory efforts of bleating the diastolic pressure was raised to $22 \mathrm{~mm}$. $\mathrm{Hg}$. Fig. 3 : this animal was under examination at a period two hours after exposure to phosgene. The mean pressures were almost the same as had been obtained from the normal animal, viz. systolic $11.5 \mathrm{~mm}$, and diastolic $1.0 \mathrm{~mm}$. When struggling occurred the diastolic pressure rose at once to $18.0 \mathrm{~mm}$. $\mathrm{Hg}$. In both these cases the same factor is no doubt responsible for the change: the diaphragm was fixed during the muscular effort and the intrathoracic pressure was raised by forcible expiration, so that the pulmonary circulation was partially obstructed, and a rise of pressure ensued.

Effects of phosgene poisoning. Tracings of the blood-pressure in the right ventricle were taken from a number of goats before and after exposure to phosgene gas. In these gassed animals the rapid and forcible respiratory movements rendered it difficult to perform the cardiac punctures successfully, and when, as occasionally bappened, there was much struggling the attempt had to be abandoned. The experiments were especially apt to fail when high doses of gas had been administered. In the following cases it was possible to carry out several successful examinations, and post mortem there was found no serious pericardial bleeding or pneumothorax, such as might have aggravated or modified the phenomena due to gas poisoning.

Experiment $D$ 30. Phosgene poisoning, with recovery. The goat was exposed to phosgene, 1 part in 5,000 by volume, for ten minutes, and soon after removal from the gas chamber it had developed severe dyspnoea, with a respirationrate of 80 per minute. The dyspnoea continued, and between twenty and thirty hours after gassing the animal passed through its most critical period, the pulserate rising to 150 per minute. Afterwards the symptoms lessened, and on the third day the respiratory rate fell to 32 per minute. Recovery had obviously set in and the animal was killed, so that the extent of the pulmonary lesion might be observed. On post-mortem examination there was found extensive oedema in the ventral half of each lung, and it was evident that oedema had been more abundant at an earlier period. The appearances were those of a gas casualty of moderate severity in process of resolution.

The tracing (Fig. 5) shows first a section taken from the normal animal at rest, and next one after exercise. Then follow nine separate sections at intervals after exposure to gas. At $1 \frac{1}{4}, 2$, and at $19 \frac{1}{4}$ hours after gassing the tracing shows rhythmical fluctuation in the blood-pressure curve, due to alterations in the cardio-respiratory ratio, the effects being similar to those of heavy exercise. The maximum systolic pressures are raised and the minimum diastolic pressures are lowered even to negative values, but the mean pressures are not very conspicuously altered: there is actually a slight diminution in the mean diastolic pressure. At 23 and at $26 \frac{1}{4}$ hours the fluctuations due to respiration are still well marked, but now there is a slight rise in the mean diastolic pressure above the normal : this coincides with the period of most critical illness. In the 

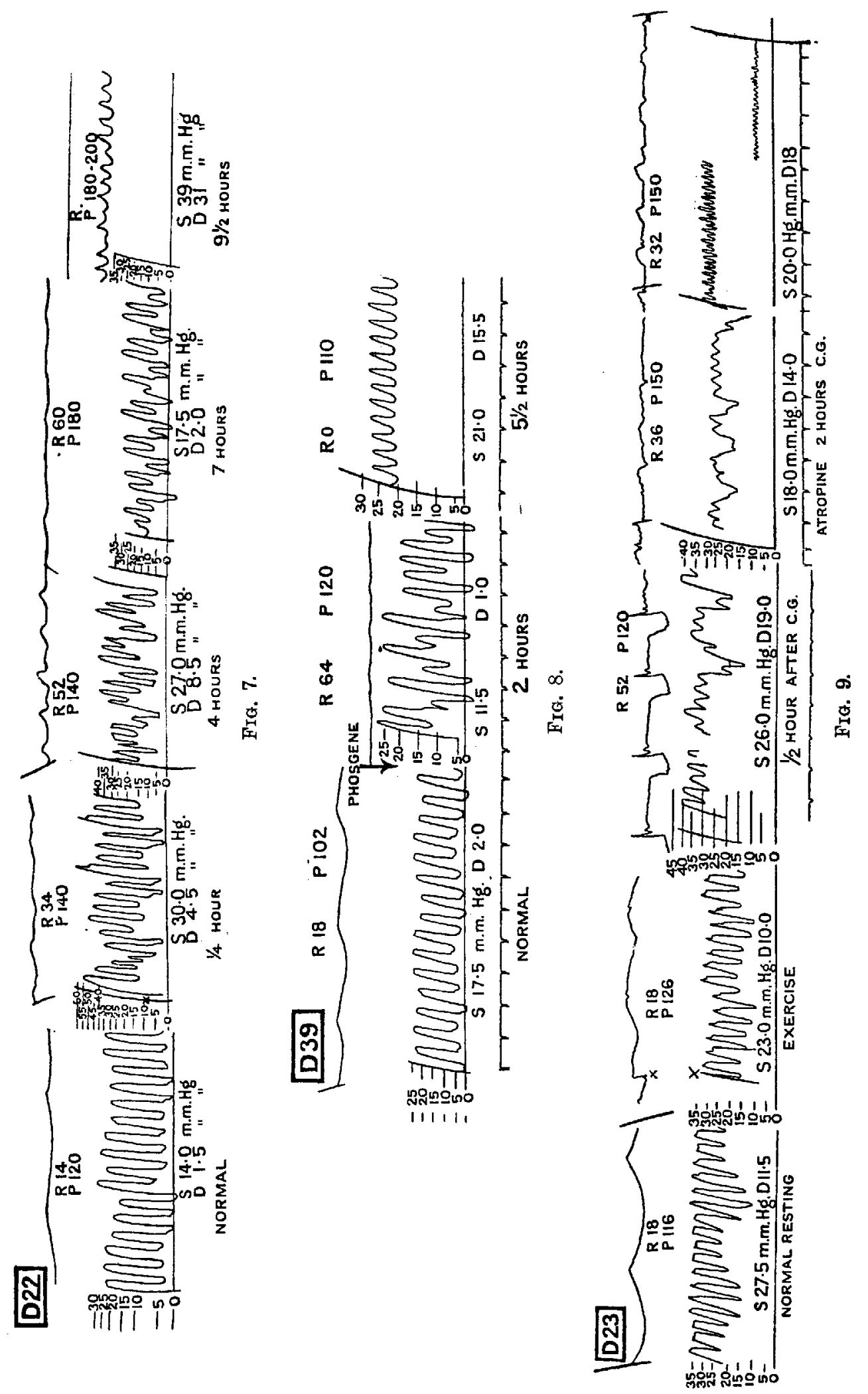
later sections of the tracings the mean diastolie pressure has fallen again, and at $66 \frac{1}{2}$ hours the blood-pressure curve is reassuming normal characters.

A series of measurements of the volume of blood circulating per minute ${ }^{\mathrm{I}}$ was made in this experiment, with the following results:

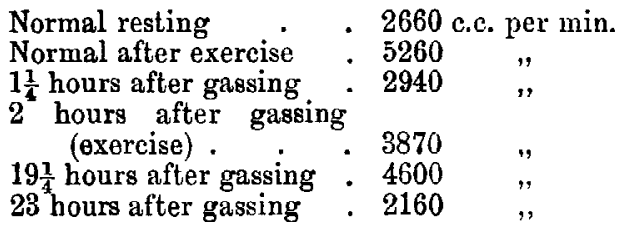

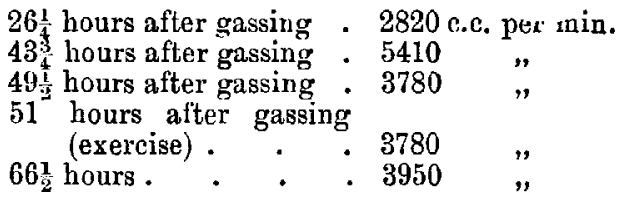

Experiment $D$ 37. Phosgene poisoning with recovery. After having been examined in the normal condition at rest, this goat was exposed to $1 / 4,000$ phosgene for ten minutes. Two hours after gassing the respiratory rate had risen to only 33 per minute from 24 . The breathing was very shallow, each respiration amounting to 120 c.c. instead of the normal of about 300 c.c. A sample of the arterial blood taken at two hours showed a severe anoxaemia, the oxygen saturation being only 57 per cent. In other goats examined two hours after the same dose of gas the arterial blood has been saturated to about 90 per cent. like the normal.

Five hours after gassing the respiratory rate had risen to 56 per minute, and the oxygen saturation of the arterial blood was 94 per cent., showing that the cause of the anoxaemia was something of a transient nature. The dyspnoea with rapid respiratory rate continued for forty-eight hours, but during this period there was no recurrence of the severe anoxaemia. On the third day the respiratory rate had fallen to 22 per minute. The animal was then killed. Post mortem the lungs were almost free from oadema and the appearances were such as to indicate that oedema had never been severe. It was considered most probable that this animal suffered from bronchiolar spasm in the early hours after gassing, with temporary sovere anoxaemia as a result.

The tracing (Fig. 6) shows a very definite rise in the mean diastolic pressure during the first twenty-four hours after gassing, but very little change in the mean systolic pressure. The influence of the increased respiratory rate on the blood-pressure curve is well marked. The section of tracing taken on the third day is approaching the normal condition.

Experiment D 22. Fatal phosgene poisoning. The goat was exposed to $1 / 5,000$ phosgene for ten minutes. The usual symptoms of dyspnoea and cardiac distress developed rapidly, and in nine hours the animal was in a critical condition and unlikely to live for more than two or three hours longer. When it was placed on its side for examination at $9 \frac{1}{2}$ hours the breathing rapidly weakened and became gasping in character, and death occurred in a few minutes. On postmortem examination the lungs exhibited severe oedema of almost universal distribution, the appearances being typical of fatal phosgene poisoning.

The tracing (Fig. 7) shows one section taken from the normal resting animal and four others at intervals after exposure to gas. A quarter of an hour after gassing there is a considerable rise in the systolic pressure, to $30 \mathrm{~mm}$. of mercury, and a slight rise in diastolic pressure. At four hours the rise of systolic pressure is maintained and the diastolic pressure is definitely raised above the normal to $8.5 \mathrm{~mm}$. Hg. Seven hours after gassing the mean pressures have fallen again almost to normal, although the condition of the animal is much worse, the pulserate being 180 per minute. At nine and a half hours the respiration has almost ceased, and there is only an occasional gasping inspiration. At this time the blood-pressure in the ventricle remains continuously at a great height, more than

1 The rate of blood flow in gas poisoning will form the subject of a subsequent communication. 
$30 \mathrm{~mm} . \mathrm{Hg}$, and there is relatively little difference between systolic and diastolic pressures.

In this instanco, coincident with a more rapid and severe clinical course, there is a definite rise in both systolic and diastolic pressures up to four hours after gassing. This probably arises from the adverse effect of the gas lesion on the pulmonary blood-vessels. The meaning of the fall in pressure at seven hours is not understood. The last section of the tracing represents a terminal phase in a moribund animal, and it is not certain that this condition of permanently raised pressure is peculiar to death from gas poisoning. The high pressure may be in part determined by diminished permeability of the pulmonary vessels, but an important factor must also be the absence of the support which is normally afforded to the pulmonary circulation by respiratory movements. An exactly parallel result has been obtained in another gassed animal in the act of death.

Experiment D 39. Fatal phosgene poisoning. The goat received a dose of $1 / 4,000$ phosgene for ten minutes. Dyspnoea developed at once and in two hours was very severe, the respirations numbering 64 per minute. At $5 \frac{1}{2}$ hours the animal was very ill and death was precipitated by turning it on its side for examination. At post-mortem examination the lungs were extremely oedematous, the condition being such as might occur in an animal dying after phosgene poisoning without being subjected to further handling.

The tracing (Fig. 8) shows a normal curve of the usual type. Two hours after gassing the curve is irregular on account of the rapid respiration, but there is no change in the mean diastolic pressure. At five and a half hours the respiration has stopped, but the heart continues to beat. As in the previous experiment the ventricular pressure is continuously very high at this examination.

These four experiments with gas poisoning show differences depending on the severity of illness in the different animals and on the times of examination. Nos. D 30 and 39 show that severe dyspnoea may be developed without the ventricular pressures being materially altered. Nos. 30,37 , and 22 show a definite rise in mean diastolic pressure at certain intervals after gassing, and in two of these the pressures subside to normal as recovery sets in. The very high ventricular pressures observed at the time of death in Nos. 22 and 39 are probably terminal only, and cannot certainly be taken to represent the conditions which existed shortly before, while the animals were still breathing. The most critical state of illness which it has been possible to examine by this method in an animal still breathing is represented in the fourth section of Fig. 9, and there the mean diastolic pressure $14 \mathrm{~mm}$. is separated by only $4 \mathrm{~mm}$. from the systolic pressure.

The work embodied in this communication was carried out at the suggestion of Mr. Joseph Barcroft, F.R.S., and under his direction. The writer has to express to him most cordial thanks for helpful advice and criticism. 